binding with the human receptor CD59 and cholesterol. To date there are no studies linking the production of VLY with the biotypes of these bacteria, which was the main objective of this study.

Methodology 90 strains of $G$. vaginalis were analysed, of which 60 were associated with normal flora and 30 with BV, biotyping was performed according to the scheme proposed by Piot et al. 1984. Dot Blot and haemagglutination evaluated VLY production and lytic capacity. The Dot Blot results were analysed densitometrically and classified as low, moderate and abundant. The lytic capacity was expressed in percentage.

Results We identified in the analysed population biotypes 1, 2, 5 and 6 of $G$. vaginalis. In the group with normal flora: biotype 1 was identified in $22 \%$, biotype 2 in $12 \%$, biotype 5 in $32 \%$ and biotype 6 in $35 \%$, while in the group associated with BV biotype 1 was identified in $33 \%$, biotype 2 in $10 \%$, biotype 5 in $10 \%$ and biotype 6 in $47 \%$. Low production of VLY was observed in 48\%, moderate in $37 \%$ and abundant in $15 \%$ of the cases in the group with normal flora. While in the group with BV low production was observed in $30 \%$, moderate in $43 \%$ and abundant in $27 \%$ of the cases. Additionally, we observed that the biotype 6 (normal flora) and 2 (BV) have the highest lytic capacity, without finding significant differences between both groups.

Conclusion We isolated the biotypes 1, 2, 5, and 6 in both study groups. The biotypes associated with VB showed higher production of vaginolysin and a greater lytic capacity. These results suggest that VLY produced by biotypes of G. vaginalis could be a key factor in the establishment and maintenance of BV.

\section{P3.134 MODELLING THE DYNAMICS OF ANTIBIOTIC RESISTANCE IN GONORRHOEA TO DETERMINE FITNESS BENEFITS AND COSTS}

${ }^{1}$ Lilith K Whittles, ${ }^{2}$ Peter I White, ${ }^{1}$ Xavier Didelot. ${ }^{1}$ NIHR Health Protection Research Unit In Modelling Methodology, Imperial College London, London, UK; ${ }^{2}$ NIHR HPRU In Modelling Methodology, Imperial College London; National Infection Service, Phe, London, UK

10.1136/sextrans-2017-053264.369

Introduction Gonorrhoea is one of the most common bacterial STI in the UK. Incidence has increased since 2008 culminating in over 41000 cases in 2015, over 50\% of which are among men who have sex with men (MSM). The bacterium has developed resistance to each frontline antibiotic in turn. Resistance to cefixime grew rapidly between its recommendation as a single-dose treatment in 2005 and removal in 2010, reaching 33\% among MSM. Since prescribing has fallen, however, so has resistance. We hypothesise there is a net fitnessbenefit to resistance when cefixime is widely prescribed but a net fitness-cost when prescriptions decline.

Methods A compartmental stochastic model incorporating latent, asymptomatic and symptomatic infection, with both cefixime-susceptible and resistant strains, was fitted to UK MSM incidence and prescription data over 2008-15 using particle Markov Chain Monte Carlo (pMCMC) methods. The fitness-cost of resistance was modelled as an increased naturalrecovery rate from asymptomatic resistant infection; the fitness-benefit was conferred when a proportion of treatmentfailures are undetected and become asymptomatic. The hypothesis was tested via 99\% credible intervals and posteriorpredictive testing.
Results We were able to replicate the data using model parameters based on literature-review. Our model suggests that natural-recovery from resistant gonorrhoea occurs 1.75 x $(99 \% \mathrm{CI}$ : 1.57-1.87) faster than from cefixime-susceptible infection, giving resistance a fitness-cost; and 91\% (99\% CI: 65\%-100\%) of treatments of resistant cases with cefixime fail, conferring a fitness-benefit when cefixime is highly-prescribed.

Conclusion The use of state-of-the-art pMCMC methods provided significant evidence in favour of our hypothesis and insights into the dynamics of cefixime-resistance in gonorrhoea. Our findings have important implications for antibiotic stewardship and public health policies, such as targeted prescriptions and combination therapy; as well as emerging resistance through similar mechanisms to the current frontline treatment, ceftriaxone.

\section{P3.135 PREDICTORS OF SEXUALLY TRANSMITTED CO- INFECTIONS IN WOMEN PRESENTING WITH BACTERIAL VAGINOSIS TO PRIMARY HEALTHCARE FACILITIES IN SOUTH AFRICA}

${ }^{1}$ Lindy Gumede, ${ }^{2} \mathrm{~T}$ Kufa-Chakezha, ${ }^{1} \mathrm{~V}$ Maseko, ${ }^{2} \mathrm{R}$ Kularatne. ${ }^{1}$ National Institute for Communicable Diseases, Johannesburg, South African Republic; ${ }^{2}$ National Institute for Communicable Diseases and University of The Witwatersrand, Johannesburg, South African Republic

\section{$10.1136 /$ sextrans-2017-053264.370}

Introduction Bacterial vaginosis (BV) is the most common cause of non-specific vaginal discharge syndrome (VDS) in South African women. BV has been implicated in the acquisition of STIs and HIV. We sought to determine STI co-infection rates and associated factors in women presenting with BV-associated VDS to primary healthcare facilities (PHCs) in South Africa.

Methods Consenting adult women, presenting with VDS to PHCs in five South African provinces, were recruited in 2015-2016. Vaginal swabs were assessed for BV using Nugent scoring; and endocervical swab-extracted DNA was tested by multiplex real-time PCR for Neisseria gonorrhoeae (GC), Chlamydia trachomatis (CT), Trichomonas vaginalis (TV) and Mycoplasma genitalium. Serum specimens were tested for HIV. Data were analysed using Stata version14 using descriptive statistics and binomial regression.

Results From 717 women enrolled, 403 (56.2\%) had BV. Of the 403 women with BV (median age 26[IQR 22-32], 201 [52.1\%] HIV positive), 215 (53.4\%) were co-infected with non-ulcerative STIs, the most common being CT [96 (23.8\%)] followed by GC [86 (21.3\%)].Univariable analysis for factors associated with STI co-infection showed a significant association with HIV infection, and a protective effect for condom use at last sex and attending a rural facility. In a multivariable model adjusting for the age, condom use at last sex, previous STI syndrome, HIV positivity and residence only age $<35$ years [relative risk (RR) 1.40 (95\% CI 1.04-1.90)] and HIV infection [RR 1.27 (95\% CI 1.06-1.52)] were independently associated with STI co-infection.

Conclusion A significant proportion of BV infected women had STI co-infections and associated risk factors. This has implications for syndromic management of VDS, and suggests that the strategy of stratification into STI and non-STI treatment groups should be reviewed. 